\title{
П.Г. КОВАДЛО ${ }^{l}$, А.Ю. ШИХОВЦЕВ ${ }^{l}$, Е.А. КОПЫЛОВ ${ }^{2,3}$, А.В. КИСЕЛЕВ ${ }^{l}$, И.В. РУССКИХ \\ ИССЛЕДОВАНИЕ ОПТИЧЕСКИХ АТМОСФЕРНЫХ ИСКАЖЕНИЙ ПО ДАННЫМ ИЗМЕРЕНИЙ ДАТЧИКА ВОЛНОВОГО ФРОНТА *
}

\begin{abstract}
Рассмотрена структура оптической турбулентности в месте расположения Большого солнечного вакуумного телескопа Байкальской астрофизической обсерватории. Приведены некоторые результаты по изучению наблюдаемых деформаций волнового фронта в целях расширения архива данных. Обсуждаются возможности выявления атмосферных слоев с повышенной интенсивностью оптической турбулентности. Даны оценки высот атмосферных турбулентных слоев в пределах нижней атмосферы.
\end{abstract}

Ключевые слова: телескоп, волновой фронт, радиус Фрида, турбулентность.

\section{Введение}

Атмосферная турбулентность существенно понижает разрешающую способность телескопов наземного базирования и ограничивает изопланатический угол атмосферной толщи. Величина, локально характеризующая интенсивность турбулентности - это структурная характеристика флуктуаций показателя преломления воздуха, входящая в «закон 2/3» Колмогорова-Обухова. Обычно в качестве интегральной характеристики интенсивности оптической турбулентности по лучу зрения принято использовать радиус Фрида [1].

Действующие и планируемые к вводу в эксплуатацию астрономические телескопы наземного базирования [2] в целях достижения их максимальной разрешающей способности в заданном поле зрения оснащаются системами адаптивной оптики разных конструкций. Эффективность коррекции изображений в части повышения пространственного разрешения при помощи системы адаптивной оптики зависит от характеристик оптической турбулентности по лучу зрения и, в частности, от средних значений и вариаций радиуса Фрида. Оптимальный выбор технических параметров адаптивной оптической системы осуществляется с учетом оптической схемы телескопа и особенностей вертикальных профилей структурной характеристики флуктуаций показателя преломления воздуха, а также профилей скорости ветра. При этом вертикальные профили метеорологических характеристик существенно изменяются как на коротких, так и на длинных промежутках времени. С этой точки зрения оценка радиуса Фрида как характеристики изрезанности волнового фронта, а также вертикальных профилей представляет интерес для астроклиматических исследований места и для оптимизации облика и технических характеристик системы адаптивной оптики.

\section{Оценка радиуса Фрида по данным измерений датчика волнового фронта. Метод определения вертикальных профилей оптической турбулентности S-DIMM+}

Для расширения архива данных о структуре оптической турбулентности и характеристик искажений волнового фронта в месте расположения Большого солнечного вакуумного телескопа (БСВТ) ведутся продолжительные оптические и микрометеорологические измерения. С помощью датчиков волнового фронта БСВТ регистрируются и архивируются гартманограммы - наборы разнесенных субизображений одного и того же объекта солнечной поверхности (поры, пятна, участки края). Совершенствуются методы измерений и изучения оптических искажений, формируемых по лучу зрения, для адаптивных систем [3]. Практически непрерывно выполняются мачтовые измерения характеристик турбулентности приземного слоя на высоте $\sim 30$ м над подстилающей поверхностью, в том числе синхронно с оптическими измерениями. Результаты изучения структуры оптической и микрометеорологической турбулентности приведены в работах [4-10].

\footnotetext{
* Восстановление турбулентных характеристик методом S-DIMM+ выполнено при финансовой поддержке соглашения № 075-15-2019-628, анализ распределения турбулентности с высотой выполнен при поддержке гранта РНФ № 19-79-00061. Данные измерений частично получены с использованием уникальной научной установки - Большой солнечный вакуумный телескоп - при поддержке ФНИ II.16.
} 
В настоящей работе рассмотрен процесс формирования искажений волнового фронта на основе анализа данных измерений дифференциального дрожания изображений с помощью датчика волнового фронта Шэка - Гартмана БСВТ, полученных летом 2018 г. Оценки радиуса Фрида по данным этих измерений подробно описаны в работе [6]. Результаты расчета радиуса Фрида, оцененного по данным измерений дифференциального дрожания изображений за 28.06.2018, приведены в табл. 1.

Таблица 1

Результаты расчета радиуса Фрида по измерениям 28.06.2018.

\begin{tabular}{|c|c|c|c|c|c|c|c|c|}
\hline Время & 14.39 & 14.41 & 14.42 & 14.44 & 14.46 & 14.50 & 14.51 & 14.52 \\
\hline$r_{0}$, см & 5.81 & 4.96 & 5.03 & 4.11 & 3.83 & 4.80 & 3.87 & 6.38 \\
\hline
\end{tabular}

Полученные значения радиуса Фрида дают представления об изменениях суммарной энергии оптической турбулентности по лучу зрения БСВТ и служат основой для анализа физической картины формирования искажений волнового фронта.

В то же время, помимо анализа интегральных характеристик оптической турбулентности, изучение ее структуры в разных высотных слоях возможно путем применения триангуляционного анализа мелкомасштабных искажений волнового фронта. Среди методов и их модификаций широко используются Slodar и S-DIMM+, основанные на регистрации и ранжировании мелкомасштабных искажений волнового фронта [10-15].

Суть метода S-DIMM+, позволяющего определять вертикальные профили оптической турбулентности по данным измерений датчика Шэка - Гартмана, а также ограничения его использования подробно описаны в [10]. В основе метода лежит связь между кросскорреляционной функцией дифференциальных смещений центров тяжести субизображений и вертикальным распределением энергетической характеристики турбулентных флуктуаций показателя преломления воздуха. Смещения центров тяжести субизображений вдоль взаимноперпендикулярных осей координат пропорциональны суммарному вкладу разных атмосферных слоев:

$$
\begin{aligned}
& C_{s x}=<\delta x_{1}(s, 0) \delta x_{2}(s, \alpha)>=\sum_{n=1}^{N} c_{n} F_{x}\left(s, \alpha, h_{n}\right) ; \\
& C_{s y}=<\delta y_{1}(s, 0) \delta y(s, \alpha)_{2}>=\sum_{n=1}^{N} c_{n} F_{y}\left(s, \alpha, h_{n}\right),
\end{aligned}
$$

где $s$ - расстояние от опорной субапертуры; $\alpha$ - угол между «осевым» и «внеосевыми» объектами; $h_{n}$ - высота турбулентного слоя $n$. Дифференциальные смещения «осевого» $(\alpha=0)$ и «внеосевого» $(\alpha \neq 0)$ объектов определяются следующим образом:

$$
\begin{gathered}
\delta x_{1}(s, 0)=\sum_{n=1}^{N} x_{n}(s)-x_{n}(0) ; \\
\delta x_{2}(s, \alpha)=\sum_{n=1}^{N} x_{n}\left(s+\alpha h_{N}\right)-x_{n}\left(\alpha h_{N}\right) .
\end{gathered}
$$

Функции $F_{x}\left(s, \alpha, h_{n}\right)$ и $F_{y}\left(s, \alpha, h_{n}\right)$ представлены в работе [10].

Коэффициенты $c_{n}$ пропорциональны интенсивности оптической турбулентности в атмосферном слое:

$$
c_{n}=5.98 D_{\text {eff }}\left(h_{n}\right)^{-1 / 3} C_{n}^{2} d h / \cos z .
$$

Поскольку для оси $y$ выражения для дифференциальных смещений имеют аналогичную форму, то для выявления особенностей в вертикальной структуре оптической турбулентности мы будем использовать анализ смещений только вдоль одной из осей.

Метод S-DIMM+ отражает подход получения данных о структуре турбулентности по изображению картины преломления, описанный в монографии [16]. В частности, рассматривая подход в широком смысле, можно считать, что если турбулентность, распределенная в некоторой турбулентной зоне, однородна и если, помимо этого, масштаб турбулентности очень мал по сравнению с поперечным сечением этой зоны, то картина распределения оптических искажений в плоскости 
апертуры должна быть эквивалентна картине распределения турбулентности в продольном сечении этой зоны. В рамках метода S-DIMM+ картина распределения турбулентности по лучу зрения оценивается по данным измерений дифференциального смещения разнесенных фрагментов субизображений, что минимизирует инструментальные искажения, вносимые вследствие ветрового обтекания телескопа.

\section{Пространственные кросскорреляционные функции дрожания изображений на Большом солнечном вакуумном телескопе}

Исследованиям волнового фронта в широком поле зрения уделяется существенное внимание при решении задач по коррекции солнечных изображений. Мы используем один из известных подходов по определению искажений волнового фронта на разных высотах, заключающийся в триангуляции локальных наклонов волнового фронта, измеренных датчиком Шэка - Гартмана. Несмотря на то, что вертикальные профили оптической турбулентности с применением анализа мелкомасштабных искажений волнового фронта восстанавливаются в среднем до высот 3-5 км $[13,17]$, на характеристики адаптивной оптики существенное влияние оказывает именно атмосферный пограничный слой.

Для определения искажений волнового фронта и вклада атмосферных турбулентных слоев на различных высотах в искажения были использованы данные измерений датчика Шэка - Гартмана, установленного в оптической схеме БСВТ. В качестве источника света было выбрано изображение края солнечного диска, анализ которого позволяет определить смещения отдельных фрагментов данного изображения, разнесенных друг от друга на разные углы. В измерениях волнового фронта поле зрения каждой субапертуры составляло 38 угл. с.

Субизображения края солнечного диска - это пространственные источники света, обеспечивающие возможность анализа отдельных фрагментов субизображений. Каждое такое субизображение в фокальной плоскости субапертуры было разбито на отдельные фрагменты, разнесенные друг от друга примерно на 1 угл. с в пределах поля зрения равного 38 угл. с. Для каждого фрагмента субизображения края солнечного диска был определен временной ряд изменений центра тяжести. Таким образом, в пределах каждой субапертуры было получено 38 временных рядов изменений центра тяжести разнесенных в пространстве фрагментов субизображений.

С помощью формулы (1) мы рассчитали пространственные кросскорреляционные функции дрожания изображений в разных субапертурах, разнесенных на расстояния 7.5 см (рис. 1), 15 см (рис. 2), 30 см (рис. 3) и 52.5 см (рис. 4). Линиями показаны пространственные кросскорреляционные функции для разных пар субапертур.

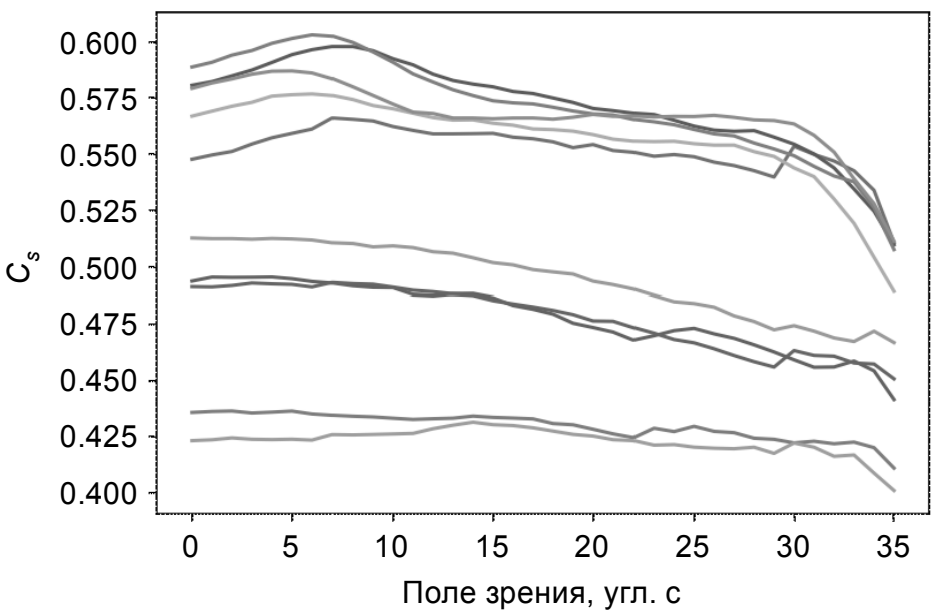

Рис. 1. Пространственные кросскорреляционные функции дрожания изображений, разнесенных на минимальное расстояние - 7.5 см. Измерения на Большом солнечном вакуумном телескопе 28.06.2018., время $14: 41$

С увеличением активной части диаметра телескопа максимум в пространственных кросскорреляционных функциях дрожания изображений должен смещаться в сторону больших углов между 


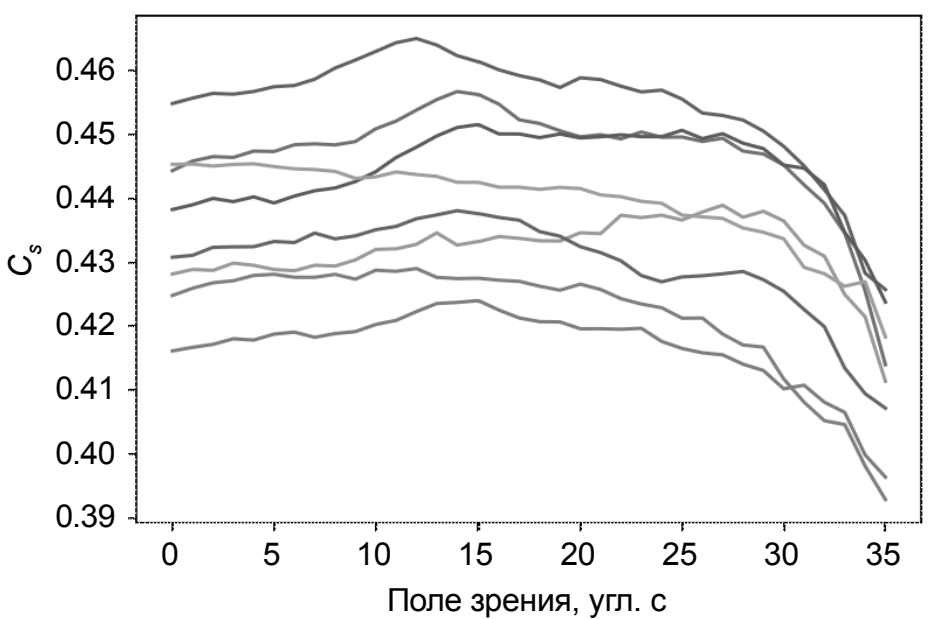

Рис. 2. Пространственные кросскорреляционные функции дрожания изображений, разнесенных на 15 см. Измерения на Большом солнечном вакуумном телескопе 28.06.2018., время 14:41

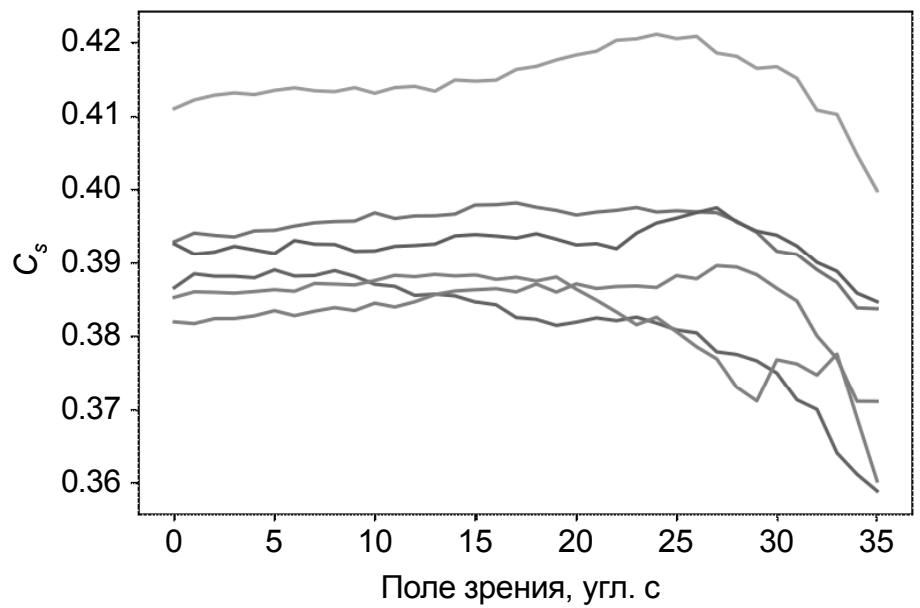

Рис. 3. Пространственные кросскорреляционные функции дрожания изображений, разнесенных на 30 см. Измерения на Большом солнечном вакуумном телескопе 28.06.2018., время 14:41

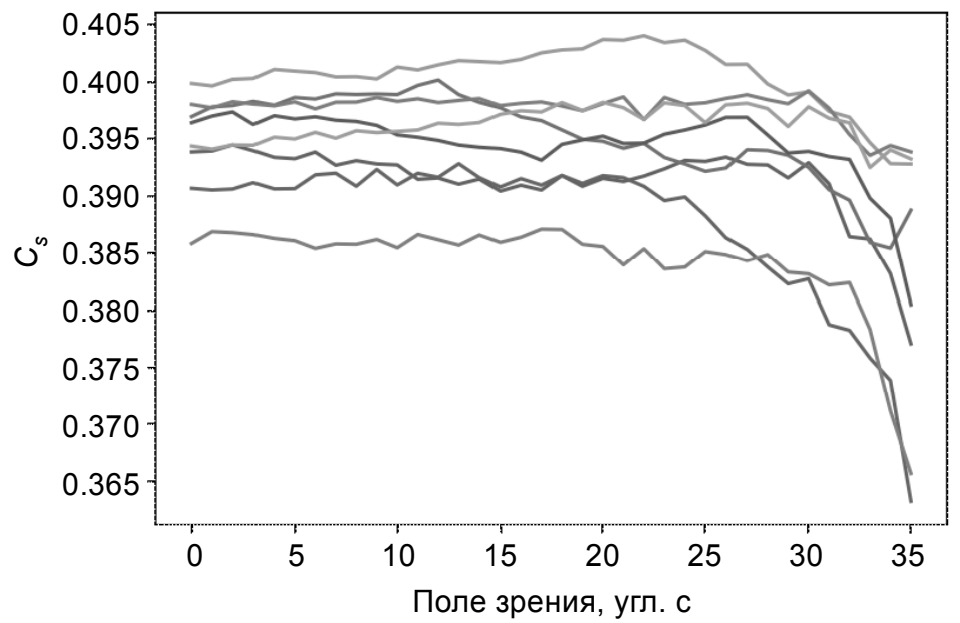

Рис. 4. Пространственные кросскорреляционные функции дрожания изображений, разнесенных на 52.5 см. Измерения на Большом солнечном вакуумном телескопе 28.06.2018., время 14:41 
«источниками» света - выбранными фрагментами субизображений солнечного края. На рис. 1-4 можно видеть это смещение. В смежных субапертурах, когда $\delta i=1$, максимум функции приходится на значения угла от 6.0 до 7.5 угл. с. С увеличением активной части диаметра телескопа он смещается на 12-15 угл. с для расстояния между центрами субапертур 15 см, при смещении 24.0-28.0 угл. с для 30 см и далее находится вне шкалы.

Для оценки профилей безразмерной характеристики турбулентности $C_{\text {норм }}=c_{n} / A$ полученные пространственные кросскорреляционные функции необходимо нормировать на корреляционную функцию $A$ в пределах опорной субапертуры, используемую в работе [18]. На рис. 5 показаны

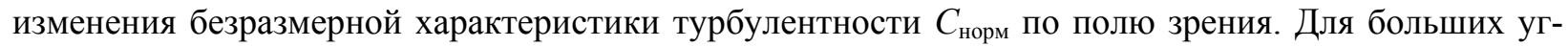
лов, соответствующих действию нижних турбулентных слоев, $C_{\text {норм }}$ превышает ее значения для малых углов.

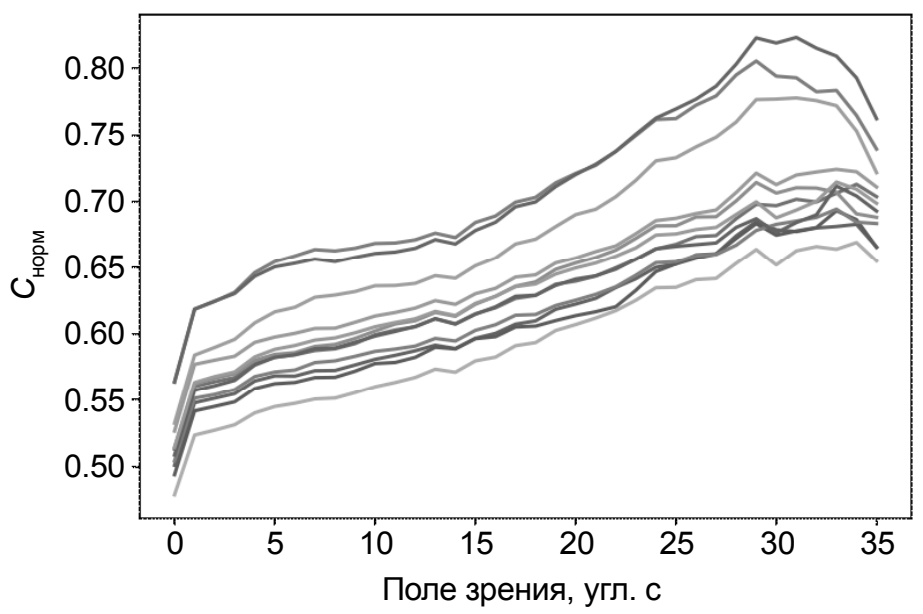

Рис. 5. Изменения безразмерной характеристики турбулентности

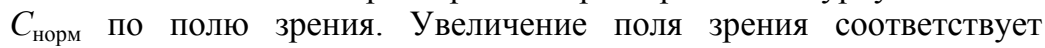
уменьшению высоты турбулентного слоя. Измерения на Большом солнечном вакуумном телескопе 28.06.2018., время 14:41

\section{Обсуждение результатов}

По данным измерений дрожаний субизображений датчиком Шэка -Гартмана мы оценили смещения положений центров тяжести в пространственных кросскорреляционных функциях. Путем триангуляции искажений волнового фронта определили высоты атмосферных турбулентных слоев, которые пропорциональны угловому смещению между фрагментами субизображений солнечного края. Результаты обобщены в табл. 2. Первая колонка соответствует расстоянию между центрами двух выбранных субапертур, вторая колонка - углу между двумя фрагментами изображения края, разнесенными на некоторый угол в пределах субапертуры, третья колонка - высоте атмосферного турбулентного слоя, который вносит искажения в пространственные кросскорреляционные функции. В четвертой колонке приведен анализ характера пространственных кросскорреляционных функций. Получено, что в месте расположения Большого солнечного вакуумного телескопа турбулентные слои наблюдаются на высотах 1150-1440 м и 570-640 м. Это находится в согласии с типичными высотами атмосферных слоев [19].

\section{Положения атмосферных турбулентных слоев, определенных по максимумам (пикам)} в пространственных кросскорреляционных функциях дрожания фрагментов субизображений солнечного края, БСВТ

\begin{tabular}{|c|c|c|c|}
\hline$D / n$, см & $\theta$, угл. с & $z$, м & Характер изменений величины $C s$ \\
\hline $7.5(\delta i=1, \delta j=1)$ & $\sim 6.0-7.5$ & $\sim 1150-1430$ & Неострый пик \\
\hline $15(\delta i=2, \delta j=2)$ & $\sim 12.0-15.0$ & $\sim 1150-1440$ & Неострый пик \\
\hline $15(\delta i=2, \delta j=2)$ & $\sim 27.0-30.0$ & $\sim 570-640$ & Неострый пик \\
\hline $30(\delta i=4, \delta j=4)$ & $\sim 24.0-28.0$ & $\sim 1230-1430$ & Неострый пик \\
\hline $52.5(\delta i=7, \delta j=7)$ & $\sim 22.0-35.0$ & $\sim 1480-2350$ & Слабый рост величины $C s$ \\
\hline
\end{tabular}


Полученные данные о пространственных кросскорреляционных функциях дрожания изображений разнесенных источников могут быть в дальнейшем использованы для расчета вертикальных профилей интенсивности оптической турбулентности, например величины структурной характеристики флуктуаций показателя преломления воздуха в режиме реального времени.

Методы восстановления профилей структурной характеристики флуктуаций показателя преломления воздуха по лучу зрения с применением дистанционных методов, основанных на данных измерений датчиков волновых фронтов, в перспективе могут быть использованы в адаптивной оптической системе расширенного поля зрения Крупного солнечного телескопа КСТ-3.

\section{СПИСОК ЛИТЕРАТУРЫ}

1. Fried D. L. // J. Opt. Soc. Am. - 1965. - V. 55. - P. 1427-1435.

2. Григорьев В.М., Дем идов М.Л., Колобов Д.Ю. и др. // Солнечно-земная физика. - 2020.T. 6. - № 2. - C. 19-36.

3. Lukin V.P., Antoshkin L.V., Bolbasova L.A., et al. // Atmospher. Ocean. Optics. - 2020.V. 33. - No. 1. - P. 85-103.

4. Ковадло П.Г., Н Й денов П. А., Шиховце в А. Ю. // Известия Иркутского государственного университета. Сер. Науки о Земле. - 2009. - Т. 2. - № 2. - С. 105-116.

5. Ботыгина Н.Н., Ковадло П.Г., Копылов Е.А. и др. // Труды Междунар. Байкальской молодежной науч. школы по фундаментальной физике и конф. молодых ученых. - 2013. - С. 303-305.

6. Botygina N.N., Kopylov E.A., Lukin V.P., et a 1. // Atmospher. Ocean. Optics. - 2014. - V. 27.No. 2. - P. 142-146.

7. Ко пы ло в Е. А., С ел и н А. А., Ш и хо в ц е в А. Ю . // Изв. вузов. Физика. - 2015. - Т. 58. - № 10/3. C. 104-106.

8. Konyaev P.A., Kopulov E.A., Lukin V.P., et a 1. // Proc. SPIE. - 2017.-V. 10466. - P. 104660N.

9. Bolbasova L.A., Shikhovtsev A.Yu., Lukin V.P., and Kovadlo P.G. // MNRAS. - 2019. - V. 482. - P. 2619-2626.

10. Th a m M. Wide field wavefront sensing: Undergraduate thesis in Astronomy. Department of Astronomy Stockholm University. - Stockholm, 2011. $-60 \mathrm{p}$.

11. Scharmer G.B. and van Werkhoven T.I. // A\&A. -2010.-V. 513.-P. A.25.

12. Шиховцев А. Ю., Киселев А.В., Ковадло П.Г. и др. // Оптика атмосферы и океана. - 2019.Т. 32. - № 12. - С. 994-1000.

13. Шиховцев А. Ю., Ковадло П.Г.,, Больбасова Л.А., Лукин В.П. // Оптика атмосферы и океана. - 2019. - Т. 32. - № 10. - С. 819-823.

14. Носов В.В., Луки н В.П., Носов Е.А., Торг ае в А.В. // Изв. вузов. Физика. - 2016. - Т. 59. № $12 / 2$. - C. $138-142$.

15. Shikhovtsev A.Yu., Kiselev A.V., Kovadlo P.G., et al. // Atmospher. Ocean. Optics. -2020. - V. 33. - No. 3. - P. 295-301.

16. Х и н ц И . О . Турбулентность. - М.: ГИФМЛ, 1963. - 680 с.

17. Shikhovtsev A., Kovadlo P., Lukin V., et al. // Atmosphere. - 2019. - V. 10. - Iss. 11. - P. 661.

18. Шиховце в А. Ю., К и селе в А. В., Ковадло П.Г.,, Р усских И.В. // Тез. докл. Междунар. конф. БШФФ-2019. - 2019. - С. 402-404.

19. Banakh V.A., Smalikho I.N., and Falits A.V.//Remote Sensing.-2020.-V. 12.-Iss. 6. - P. 955.

\footnotetext{
${ }^{1}$ Институт солнечно-земной физики СО РАН, г. Иркутск, Россия

${ }^{2}$ Институт проблем лазерных и информационных технологий РАН филиал Федерального научно-исследовательского центра «Кристаллография и фотоника» РАН, г. Шатура, Московская область, Россия

${ }^{3}$ Институт оптики атмосферы им. В.Е. Зуева СО РАН, г. Томск, Россия
}

\footnotetext{
Ковадло Павел Гаврилович, д.ф.-м.н., ст. науч. сотр. ИСЗФ СО РАН, e-mail: kovadlo2006@rambler.ru;

Шиховцев Артем Юрьевич, к.ф.-м.н., науч. сотр. ИСЗФ СО РАН, e-mail: Ashikhovtsev@iszf.irk.ru;

Копылов Евгений Анатольевич, к.ф.-м.н., ст. науч. сотр. ИПЛИТ РАН - филиал ФНИЦ «Кристаллография и фотоника» РАН, науч. сотр. ИОА СО РАН, e-mail: kea@iao.ru;

Киселев Александр Викторович, мл. науч. сотр. ИСЗФ СО РАН, e-mail: kiselev@iszf.irk.ru;

Русских Иван Викторович, инженер-програмист ИСЗФ СО РАН, e-mail: vanekrus@iszf.irk.ru.
} 